\title{
"Imperfektibles" sprachliches Wissen Theoretische Vorüberlegungen zu "sprachlichen Zweifelsfällen"
}

\author{
Gerd Antos (Halle a. d. Saale)
}

\begin{abstract}
In linguistic cases of doubt a differentiation has to be made between the pragmatic reflections of language by laypersons (SZ 1) and the common 'extra-communicatice' reflections by linguists (SZ 2). These structural inhomogeneities of languages are based upon (e.g. orthographic) variants, 'openings', so called 'language fluctuations' or some other irregular forms that have developed historically (SZ 2 = 'objective imperfectibility' of the linguistic system). On the other hand, the competence theoretical notion of 'linguistic doubt' (of the type SZ 1) requires that speakers become aware or are aware of their lacking, constricted, or insecure knowledge in their language usage (= SZ 1: 'subjective imperfectibility'). From a point of linguistic development, both linguistic cases of doubt can be seen as indicators for potential 'mutants' in the inheritance of linguistic forms, structures and functions. Hence, 'imperfectibility' (both as a quality of a system and as a cognitive substrate of it) is an important condition for future linguistic change.
\end{abstract}

\section{$1 \quad$ Einleitung}

Kaum ein Phänomen ist in der "klassischen" linguistischen Diskussion so marginalisiert worden wie sprachliche Zweifelsfälle. Die Exkommunikation von realen Sprechern oder Schreiberinnen aus der strukturalistischen Linguistik spielte dabei ebenso eine Rolle wie die Vorstellung, dass der native speaker im Prinzip in der Lage sein müsse, dichotomisch unterstellte Grammatikalitätsurteile abzugeben. Hinzu kam wohl auch, dass sprachliche Zweifelsfälle Gegenstand sprachideologischer Auseinandersetzungen waren (Klein 2003b). Dass sie bis heute im Zusammenhang mit der sozialen Durchsetzung und Sanktionierung orthographischer oder stilistischer Normen gesehen werden, macht die analytische Reflexion über sie schließlich nicht einfacher. Die Konsequenz: Sprachliche Zweifelsfälle wurden lange Zeit von einer sich vor allem als theoretisch verstehenden Linguistik bestenfalls als Performanzphänomene und das heißt: als Epiphänomene betrachtet, die - wenn überhaupt - in der "Laienlinguistik" (Antos 1996) eine Rolle spielen.

Erst mit dem Erfolg der Soziolinguistik (Labov) wurde klar: Typisch für den sprachlichen Haushalt einer Gesellschaft ist über den Standard hinaus eine Fülle von sprachlichen Varietäten, die mit ihren dialektalen, stilistischen oder fachsprachlichen Variantenreichtum das Formulieren häufig schwer machen. Mit der "Empirisierung der Linguistik" und dem 
Studium großer Datenmengen wurde dann spätestens seit den 80er Jahren klar, dass der konkrete Sprachgebrauch weitaus vielgestaltiger und normunabhängiger ist, als dies standardorientierte Vorstellungen von Sprache nahe legen. Phänomene wie Versprecher, Fehlstarts oder Korrekturen aller Art ("repairs") wurden nicht mehr nur als sprachliche Defizienzen betrachtet. Vielmehr boten solche Phänomene nun einen unverhofften Blick in dezidiert theoretische Fragestellungen - sei es aus einer kognitivistischen, einer grammatischen oder einer gesprächsanalytischen Perspektive.

Im Folgenden soll versucht werden, Vorüberlegungen $\mathrm{zu}$ einer theorieorientierten Betrachtung von sprachlichen Zweifelsfällen anzustellen. Zunächst soll gezeigt werden, dass man bei sprachlichen Zweifelsfällen eine von Laien betriebene "kommunikationspragmatische" Reflexion über Sprache (SZ 1) von einer in der Linguistik üblichen "extrakommunikativen" (Gerold Ungeheuer) Reflexion (SZ 2) unterscheiden muss, die die sprachstrukturellen Inhomogenitäten einer Sprache thematisiert. Natürlich basieren auch viele Zweifelsfälle in konkreten Kommunikationskontexten (SZ 1) auf sprachstrukturellen Varianten, Lücken, auf sog. "Sprachschwankungen" oder auf anderen sprachhistorisch entstandenen unregelmäßigen Formen. Die daraus resultierenden sprachstrukturell bedingten sprachlichen Zweifelsfälle sollen als SZ 2 gesondert betrachtet werden. Darüber hinaus gibt es aber weitere Zweifelsfälle (SZ 1), die z.B. durch Konkurrenzen zwischen Standard und Substandard ebenso hervorgerufen werden können wie durch Konflikte zwischen verschiedenen Varietäten oder zwischen unterschiedlichen (z.B. Rechtschreib-)Normen.

Ausgehend von diesen Differenzierungen soll dann versucht werden, einen theoretischen Zugang zu dem schillernden Phänomen zu suchen. Dies auch deshalb, weil uns "sprachliche Zweifelsfälle" von Sprechern oder Schreiberinnen einen Einblick in das vermitteln können, was in der Linguistik unter Konzepten wie "sprachliche Kompetenz", "linguistic awareness" (Sprachbewusstheit) oder "Sprachgefühl"1 diskutiert wird.2 Dabei sollen insbesondere drei Thesen diskutiert werden:

"Sprachliche Zweifelsfälle" i. S. (SZ 1) beruhen auf einem fehlenden, eingeschränkten oder unsicheren Wissen von Sprechern/Schreiberinnen (oder bestimmten Sprechergruppen) über die sozial akzeptierte Korrektheit oder Angemessenheit der geplanten oder verwendeten Formulierung für bestimmte kommunikative Aufgaben.

Ein Wissen über disparate Formen und Strukturen im sprachlichen Haushalt einer Gesellschaft und damit auch ein Wissen über Grenzen sprachlich-kommunikativen Wissens basiert auf der Annahme, dass sowohl die heterogenen Systeme in einem sprachlichen Haushalt als auch die darauf beruhenden Kompetenzen von "Sprecher/Hörer" einerseits disparat, aber andererseits auch im Hinblick auf Veränderungen "offen" sind. Sprachliches Wissen ist (wie jedes komplexes Wissen) daher "imperfektibel"!

\footnotetext{
${ }^{1}$ Gauger, H.-M. u.a.(1982): Sprachgefühl?. Ist Berufung auf "Sprachgefühl" berechtigt? Antworten auf die Preisfrage der Deutschen Akademie für Sprache und Dichtung vom Jahre 1980. Heidelberg

2 Eine besonders extreme Position dazu ist Paul Hoppers "Emergent Grammar Hypothesis" (Hopper 1987, Weber 1997).
} 
Unter einer sprachevolutionären Sichtweise können "sprachliche Zweifelsfälle" als Indikatoren für mögliche "Mutanten" in der Vererbung sprachlicher Formen, Strukturen und Funktionen aufgefasst werden. Wären sprachliche Systeme in sich vollkommen homogen und im Hinblick auf andere Schnittstellen definit (und nicht überlappend oder konkurrierend), dann gäbe es auch keinen Sprachwandel. Imperfektibilität (als Systemeigenschaft wie als ein kognitives Substrat davon) ist daher eine entscheidende Bedingung für zukünftigen sprachlichen Wandel.

Auf dem Hintergrund dieser Thesen erscheinen sprachliche Zweifelsfälle nun nicht mehr als Epiphänomene von Sprachstrukturen oder eines individualistisch gedachten Sprachgebrauchs, sondern als wichtiger Motor für eine synchrone wie diachrone Sprachdynamik.

\section{Vorüberlegungen}

Im Hinblick auf die nach Umfang und Formenreichtum kaum überschaubaren sprachlichen Zweifelsfälle sollten zwei Konzepte unterschieden werden: Im öffentlichen Bewusstsein dominiert bei sprachlichen Zweifelsfälle die von Laien betriebene "kommunikationspragmatische" Reflexion über Sprache (SZ 1). Diese muss von einer in der Linguistik aufkommenden "extrakommunikativen" (Gerold Ungeheuer) Reflexion unterschieden werden (SZ 2). Letztere wird vorzugsweise von Sprachwissenschaftlerinnen und Sprachwissenschaftlern betrieben und bezieht sich im Wesentlichen auf systemimmanente, zumeist grammatische bedingte Zweifelsfälle.

Diese Unterscheidung hat eine Reihe von Konsequenzen, die hier nur angedeutet werden sollen: Laien geraten bisweilen dann ins Zweifeln, wenn sie z.B. über die orthographische Richtigkeit oder über die stilistische Angemessenheit bei der Wahl bestimmter Formulierungen unsicher werden. Wenn sie es können, konsultieren sie dann eine Sprachberatung oder nehmen zum Beispiel den DUDEN: "Richtiges und gutes Deutsch. "Wörterbuch der sprachlichen Zweifelsfälle" (Bd. 9) 3 in die Hand. Dahinter steht der Wunsch nach einem sozial prämierten ,korrektem Deutsch' bzw. die Sorge sich nicht zu blamieren. Diese "laienlinguistische Reflexion" resultiert also primär aus Unsicherheiten über soziale Anforderungen an eigene oder fremde Kommunikationsbeiträge. Solche "laienlinguistischen" Zweifel lassen dann sehr schnell nach Instanzen von Sicherheiten rufen. In diesem Sinne bedingen sich "sprachliche Zweifelsfälle" und Wünsche nach "korrektem Deutsch" in gewisser Weise wechselseitig.

Auf der Grundlage solcher Beobachtungen mag es nahe liegen, "sprachliche Zweifelsfälle" als "Performanzphänomene" einzuordnen, d.h. als Phänomene, die nichts mit Sprache, wohl

\footnotetext{
${ }^{3}$ Früher hieß er: "Die Zweifelsfälle der deutschen Sprache". Der DUDEN weist - wie in den früheren Auflagen auch - darauf hin, dass dem Band 9 Tausende von Anfragen an die Sprachberatungsstelle der Dudenredaktion zu Grunde liegen. Die Beachtung und Beobachtung von tatsächlich geäußerten "sprachlichen Zweifelsfällen" - so darf man daraus schließen - verdanken sich daher nicht unwesentlich massiver laienlinguistischer Intervention (Antos 1996).
} 
aber mit bestimmten Umständen ihrer Verarbeitung, insbesondere mit situativen, medialen, sozialen usw. Umständen ihrer sprecherischen oder schreiberischen Produktion zu tun haben.

Eine solche Position übersieht jedoch sowohl die quantitative "Massivität" des Phänomens ebenso wie seine Funktion: Nach Klein (2003b:9) müsste man bereits auf der Grundlage des einschlägigen, knapp tausendseitigen DUDEN-Bandes mit ca. 5000 Zweifelsfällen rechnen. Es scheint aber ziemlich unsinnig zu sein, eine solche Fülle einzelsprachlicher Phänomene, die zudem lexikonartig auflistbar sind, als bloße "Performanzphänomene" etwa Wortfindungsschwierigkeiten, "Reparaturen" oder Konzentrationsschwächen beim konkreten Formulieren an die Seite stellen zu wollen. Vielmehr verbergen sich hinter solchen und vielen anderen "subjektiven" Manifestationsformen ganz unterschiedliche Phänomene und sprachlich-kommunikative Probleme (Antos 1996):

Die meisten Sprecher oder Schreiberinnen würden das Auftreten von "sprachlichen Phänomenen" auf Befragen mit Anforderungen an die Schriftlichkeit in Zusammenhang bringen, insbesondere mit Anforderungen und Sanktionsdrohungen, die aus Rechtschreibund Stilnormen resultieren. Dass "sprachliche Zweifelsfälle" offenkundig als ein kollektives Problem aufgefasst werden, zeigen vielfältige Angebote einer entsprechenden Beratungsliteratur ebenso wie eine Reihe von Sprach- und Grammatikberatungsstellen. ${ }^{4}$ Zudem ist das Phänomen - so scheint es - tief in dem öffentlichem Sprachbewusstsein verankert. $5^{5}$

Kurz: "Sprachliche Zweifelsfälle" äußern sich zwar subjektiv als Probleme von Sprechern oder Schreiberinnen. Sie beruhen aber in ihrer Mehrzahl, vor allem aber im Hinblick auf ihre Typik auf objektiven Problemen. ${ }^{6}$ Diese resultieren u.a.

- aus dem Bewusstsein von Normen und Sanktionen, die mit der Schriftlichkeit in literalen Gesellschaften verbunden sind;

- aus der "Mehrsprachigkeit in der Muttersprache", also aus sozialen, stilistischen usw. Variationen und Varietäten ("Heterogenitätsthese") und

- aus systembedingter grammatischer Disparatheit (Lücken, Brüche, Interferenzen, falsche Analogieformen, Form- und Strukturkonkurrenzen (z.T. als Ergebnis unterschiedlicher Grammatikalisierungsprozesse) usw.).

Man sieht: Hinter der Bezeichnung "sprachlicher Zweifelsfall" verbergen sich vielfältige und äußerst heterogene Phänomene und Probleme der Grammatik, der soziolinguistischen Ausdifferenzierung einer Sprache und schließlich des Sprachgebrauchs. Daher scheint die

\footnotetext{
${ }^{4}$ Z.B. Sprachberatungsstellen in Mannheim (DUDEN), des Bertelmann Lexikon Verlags in Gütersloh oder bei der GfdS in Wiesbaden - neben denen an der Universität Halle. An der Universität in Potsdam gibt es zudem ein "Grammatik-Telefon"; vgl. Bünting/Pospiech (1996), Höhne (1990) ders. 1991a, Biere/Hoberg (ed.) (1995).

5 So stellt Klein mit Blick auf die Geschichte der Reflexion über Sprache zurecht fest: "Bei vielen Zweifelsfällen existiert eine machtvolle Tradition der metasprachlichen Thematisierung" (Klein 2003a: Thesen, Klein 2003b).

6 "Weil natürliche Sprache historisch wandelbar ist, weil sie aus einer komplexen Varietätenvielfalt besteht und ihr System zudem notwendigerweise gewisse Lücken und Brüche umfasst, ist die Existenz von Zweifelsfällen immer schon vorprogrammiert" (Klein 2003b:10).
} 
angedeutete Unterscheidung in SZ 1 und SZ 2 sinnvoll: Der kompetenztheoretisch angelegte Begriff SZ 1 hat eine andere, nämlich eine "kommunikationspragmatische" Funktion als der extrakommunikative Begriff SZ 2. Zwar besteht ein Zusammenhang zwischen beiden: SZ 2 ist zwar einer der Gründe für sprachliche Zweifelsfälle von Sprechern und Schreiberinnen (SZ1), aber eben nicht der alleinige.

\section{Gründe für grammatisch bedingte sprachliche Zweifelsfälle}

\subsection{Sprachstrukturelle Disparatheit}

SZ 2 beruhen auf objektiven sprachlichen Phänomenen, die zu Formulierungsproblemen führen. Sie resultieren aus einer sprachhistorisch entstandenen und sich systematisch manifestierenden sprachstrukturellen Disparatheit von Varietäten/Sprachen. Dahinter steht die schlichte Erkenntnis, dass Sprachen (und Sprachvarietäten) nicht nur unterschiedlich reichhaltig, sondern auch verschiedengestaltig und lückenhaft (vgl. die Wortfeld-Theorie) sind, insbesondere: dass Sprache(n) nicht strukturell homogen sind. Die Aufdeckung von sprachstruktureller Disparatheit fördert insbesondere die Erkenntnis von miteinander konkurrierenden grammatischen Regeln, von Regelkonflikten und sprachhistorisch bedingten Abweichungen. Solche sprachstrukturell bedingten Disparatheiten analytisch zu problematisieren und zu klären, ist eine wichtige Aufgabe der Grammatikforschung. Diese extrakommunikative Reflexion über sprachstrukturelle Disparatheiten kann im Sinne von SZ 2 bei Grammatikern unter einer kompetenztheoretischen Perspektive dazu führen, Formen und Strukturen von sprachlicher Inhomogenität als "Zweifelsfälle" zu konzeptualisieren.

\subsection{Heterogenität von Varietäten}

In einer elaborierten Sprachgemeinschaft beherrschen die meisten Sprecher bzw. Schreiberinnen immer mehrere Stile und Register. Hinzutreten können Dialekt, Soziolekt oder Fach- und Sondersprachen. Diese "Mehrsprachigkeit in der Muttersprache" hat William Labov zur "Heterogenitätsthese" verdichtet: Danach bestehen Sprachen aus mehreren Varietäten (z.B. Dialekten) zumeist auch aus mehreren Varianten! Varietäten einer Sprache fallen in Umfang, Akzeptanz (Kilian 2003) und in ihrer kommunikativen Reichweite zum Teil sehr unterschiedlich aus. Sie haben hinsichtlich Lautung, Lexik und in bestimmten Grenzen auch hinsichtlich ihrer Morpho-Syntax ihr eignes Profil. Wie aus der Aufzählung der verschiedenen Varietäten deutlich wird, können sie einerseits zusammenwirken (z.B. bestimmte Soziolekte, Stile, Register), andererseits schließen sie sich im Hinblick auf bestimmte Stile oder Normen aus (z.B. die Kombination von Dialekten, Standard oder Stilen). Die Kenntnis und Berücksichtigung bestimmter - häufig in Opposition stehender - Varietäten, insbesondere von Stiloppositionen ist nach Labov konstitutiv für sprachliche Kommunikation. Das dafür benötigte Wissen hinsichtlich Anwendungssituationen, Grenzen und Nuancen von Sprachen (samt Varietäten) kennzeichnet zugleich die soziokommunikative Stellung von Sprechern (und Schreiberinnen).

Aus der Vielzahl und Heterogenität von Varietäten, insbesondere aus der Konkurrenz zwischen ihnen (und Varianten) resultieren für Sprecher oder Schreiberinnen in konkreten 
Kommunikationssituationen fast notwendigerweise "sprachliche Zweifel". Insbesondere gilt: Je mehr Stile, Register und sonstige Varietäten und Varianten jemand beherrscht, um so mehr Zweifelsfälle sind tendenziell wahrscheinlich. Dies resultiert nicht nur aus zu unterstellenden Wissensgrenzen von Sprachhandllungsteilnehmer (siehe unten).

Nach Feilke $(1994,1996)$ ist die Kenntnis sprachlicher Nuanciertheit sogar fundamental für die umfassende Beherrschung einer Sprache ("Commen sense-Kompetenz"): Dahinter steht die Auffassung, dass sich sprachliches Wissen nicht nur auf Formen und Strukturen, sondern auf typische Gebrauchskontexte erstreckt und sprachliche Kompetenz daher im hohen Maße ein kontextspezifisches Wissen impliziert. Dies zeigt sich besonders deutlich an lexikalischen, phraseologischen oder stilistischen Varianten, deren gebrauchsspezifisch unterschiedliche Reichweite ebenso Formulierungsprobleme aufwerfen kann wie eine stilistisch "stimmige" Kombination von im Prinzip akzeptablen sprachlichen Formen. Kurz: Sprachliche Heterogenität ist in etwa das, was auch und insbesondere Fremdsprachenlerner vor erhebliche Schwierigkeiten in der zu lernenden Sprache stellt.

\subsection{Rechtschreibnormen}

Wer Sprecher oder Schreiberinnen auf prototypische Zweifelsfälle anspricht, wird insbesondere nach der Rechtschreibreform - in der Regel auf Probleme der Orthographie verwiesen werden. Dies gilt zum einen sowohl für Unstimmigkeiten zwischen verschiedenen Regelwerken ${ }^{7}$ als auch für Inkonsequenzen innerhalb eines bestimmten Regelwerks. Ferner werden jetzt weitaus mehr Varianten (Groß- bzw. Kleinschreibung, Getrennt- bzw. Zusammenschreibung, Fremdwortschreibungen usw.) zugelassen als früher. ${ }^{8}$ Schließlich gibt es noch immer ein Nebeneinander, genauer: eine mitunter ideologisch aufgeladene Konkurrenz von alter und neuer Rechtschreibung. ${ }^{9}$ Klar ist: All das führt bei vielen selbst versierten Schreibern zu einer fortwährenden Verunsicherung im Hinblick auf eine normgerechte Schreibung.

\subsection{Fazit}

Objektive Grundlage von sprachlichen Zweifelsfällen sind drei ganz unterschiedliche Formen von Disparatheiten: 1. eine sprachhistorisch ablesbare sprachstrukturelle Disparatheit, 2. eine komplexe Varietätenheterogenität und 3. Regelkonkurrenzen in der Orthographie. Sie alle sind Ressourcen für objektive sprachliche Zweifel im Sinne von SZ 2. Allerdings wäre es falsch, diesen Zusammenhang deterministisch zu sehen. Sprachhistorische Entwicklungen, die zu den genannten Disparatheiten führen, können, müssen aber nicht von Sprechern und Schreiberinnen bemerkt werden. D.h. sie können, müssen aber nicht zu sprachlichen

\footnotetext{
${ }^{7}$ Der neue DUDEN schreibt Leid (R 46) in: Es tut mir Leid groß. Vertreter der alten Rechtschreibung wie etwa Theodor Ickler geißeln dies als grammatische falsche Schreibweise (Ickler 2000: 10).

${ }^{8}$ Die teilweise neu geregelte Differenzierung zwischen ss und $\boldsymbol{\beta}$ im Deutschen wird bekanntlich nicht von der Schweiz übernommen, die weiterhin kein $\boldsymbol{\beta}$ kennt. Dies ist ein Hinweis darauf, dass auf ein einheitliches Regelwerk für die deutschsprachige Orthographie verzichtet wurde.

${ }^{9}$ So führt z.B. die FAZ die alten Rechtschreibung weiter.
} 
Zweifelsfällen führen. Wie Ulrich Schmitz zeigt, findet man auch und gerade in der Schriftsprache "fehlerhafte" Formen, die belegen, dass sprachhistorische Veränderungen keineswegs von den sprachliche Handelnden bemerkt werden. ${ }^{10}$

\section{$4 \quad$ Imperfektibilität}

Sprachen samt sprachlicher Varietäten sind immer Entwicklungsformen "im Fluss". Diese sprachhistorische Binsenweisheit stößt sich allerdings in einer gewissen Weise mit der strukturalistischen Modellierung von "Sprache als System" und dem Eindruck, dass - lässt man einmal die literarische (Hoffmansthal), philosophische (Wittgenstein) oder politisch motivierte Sprachkritik beiseite - Sprache(n) ein taugliches Mittel für Kommunikation ist. Trotzdem zeigen die skizzierten sprachstrukturellen Disparatheiten, die Varietätenvarietäten und -variationen sowie Regel- und Normenkonkurrenzen aller Art, dass Sprachen "unvollendete" Gebilde sind. Das heißt zum einen: Sie sind keineswegs perfekt, ein Wunsch, der z.B. im "Wiener Kreis" als Idealsprachen-Programm diskutiert wurde. Dies zeigt sich nicht zuletzt an Varianten, an Konkurrenzformen, an Lücken und an Brüchen, kurz an all jenen "Unregelmäßigkeiten" und "Inkonsequenzen",11 die für lebendige Sprachen so typisch sind. Das hat zum anderen aber auch einen großen Vorteil: Sprachen sind hinsichtlich ihrer lautlichen, lexikalischen und morphosyntaktischen Aspekte "für die Zukunft hin offen". Nicht zuletzt diese "imperfektiblen" Systemeigenschaften ermöglichen Sprachwandel, ja treiben ihn bisweilen voran. Dies selbst dann, wenn unter sprachpolitischen Aspekten Sprachwandel gerade verhindert werden soll (z.B. durch Institutionen wie der Académie francaise).

Angesichts der Tatsache, dass perfekte Systeme weder in der Natur, noch in Gesellschaften (über-)lebensfähig sind, mag die Erkenntnis, dass auch Sprachen "imperfektibel" sind, banal erscheinen. Wahrscheinlich würde man gar nicht auf einen solchen Gedanken kommen, wenn nicht solche Idealvorstellungen wesentlich durch eine seit der Aufklärung einsetzenden Sprachstandardisierung, -vereinheitlichung und durch das Aufkommen einer breiten Schriftlichkeit gefördert worden wären. ${ }^{12}$ Die These, dass Sprachen sowohl sprachhistorisch

10 Im Hinblick auf schriftsprachliche Beispiele wie: "Käpt'n Blaubär trifft den größten Teddybär der Welt" (Schlagzeile auf einem Plakat der Messe Essen, Oktober 1999) stellt Schmitz fest: "Seit weit mehr als tausend Jahren ist die deutsche Sprache dabei, ihren Sprachbau vom synthetischen zum analytischen Typ zu verändern, d.h. die Sprecher machen immer weniger Gebrauch von Flexion. Viele einzelne Erscheinungen tragen zum Flexionsschwund bei: Wegfall von Kasusendungen beim Substantiv, Ersatz von Kasusflexion durch präpositionale Fügungen, Verwendung unflektierter Appositionen statt flektierter Attribute, Abbau der starken Verbkonjugation und des Vokalwechsels, Rückgang des flexivischen Konjunktivs und sein Ersatz durch das Verbgefüge mit würde, Ersatz synthetischer Tempora durch periphrastische Konstruktionen, analytische (mit Modalverben gebildete) Verbmodi u.a." (Schmitz 2000:135)

11 Schon Leibniz und andere Gelehrte der Aufklärung versuchten, "zweifelhafte Fälle durch bezug auf die Vernunft zu lösen." Man baute darauf, "dass derjenige, der logisch und vernünftig nachdenkt, ein für alle Mal vom sprachlichen Zweifel befreit ist" (Klein 2003b:5).

12 "Varianten, Doppelformen und Sprachschwankungen sollten in einer kollektiven gesellschaftlichen Anstrengung aus der Sprache getilgt werden, weil sich darin die noch unvollendete Standardisierung des Deutschen verkörperte. Wer zweifelt, ist sozusagen in den Schranken seiner Individualität und regionalen 
als auch synchron betrachtet trotz aller sprachplanerischen Versuche keine homogenen Gebilde sind/werden, hängt also ersichtlich von dem Stand der kollektiven wie individuellen Sprachbewusstheit und damit vom kulturell beeinflussten Wissens- und Reflexionshorizont ab. Damit verlassen wir eine im Kern verdinglichende Betrachtung von Sprache. Diese billigen Sprache einen Objektcharakter zu bzw. einen reifizierenden Status und wechseln zu einer kompetenztheoretischen Betrachtung von Sprache, wie sie vor allem in der kognitiven Linguistik (Chomsky, Langacker, Lakoff) üblich geworden ist.

Dieser Wechsel ist wichtig: Ob es im Deutschen "der Friede" oder "der Frieden" heißen muss (Beispiel Klein 2003b) oder "Hallenser" (Bewohner der Saalestadt) bzw. "Haller" (Bewohner einer Stadt im Münsterland) ist nur schwer dem "System des Deutschen" zuzuschreiben. Vielmehr hängt es davon ab, was bestimmte Sprecher bzw. Schreiberinnen in lexikalischer oder sprachgeographischer Hinsicht von der deutschen Sprache wissen. Auf einem solchen Hintergrund erst kann das formuliert werden, was die "Imperfektibilitäts-These" genannt werden soll. Sie besagt, dass zum Wissen über Sprache und ihren Gebrauch auch und nicht zuletzt ein Wissen über sprachlich-kommunikative Unklarheiten, Lücken, Grenzen gehört bzw. ein Wissen über mögliche (ästhetische, kreative oder rhetorische) Spielräume (beispielsweise in der Laut- oder Wortbildung). In diesem Sinne beruhen "sprachliche Zweifelsfälle" i. S. (SZ 1) auf einem fehlenden, eingeschränkten oder unsicheren Wissen von Sprechern oder bestimmten Sprechergruppen über die verwendeten (mündlichen oder schriftlichen) Sprachformen.

Dieses imperfektible Sprachwissen beruht ganz wesentlich, aber nicht ausschließlich auf den genannten sprachstrukturellen Inhomogenitäten. Formen wie Das König der Biere oder Da werden sie geholfen oder Laut und Luise beruhen nicht auf den genannten Disparatheiten des Typs SZ 2. Sie sind aber auch nicht schlichte "Fehler", so wenig wie die Schreibung von BahnCard oder die selbstbewusste Verwendung des Wortes Mobile für Handy. Trotzdem provozieren sie bei vielen sprachliche Zweifel im Sinne von SZ 2. Womöglich erst in einem (bewusst angestoßenen) Reflexionsprozess wird deutlich, dass jenseits individueller oder gruppenspezifischer Beschränkungen Wissen über Sprache ein "offenes" Wissen impliziert.

Dieses imperfektible Wissen zeitigt eine ganze Reihe von zum Teil überraschenden Konsequenzen:

Anders als extrakommunikative SZ 2 entzünden sich SZ 1 beim konkreten Formulieren und Verstehen.

Anders als SZ 2 sind diese Zweifel (SZ 1) sanktionsbedroht, d.h. sie können Auswirkungen auf die soziale Bewertung oder Rolle des Sprechenden oder Schreibenden haben ("LübkeDeutsch").

Bei SZ 1 und SZ 2 ist partiell das anders geregelt, was man unter Fehler versteht. "Der Butter, das Nicole, hat gebrungen sind Fehler im Standarddeutschen. In der Pfalz oder im Saarland sind sie gebräuchlich, können aber für viele Dialektsprecher zu sprachlichen Zweifelsfällen

Herkunft befangen und noch nicht vollständig in die vernünftige Homogenität der deutschen Kommunikationsgemeinschaft integriert." (Klein 2003b:5). 
führen. Im Hinblick auf die gesamte deutschsprachige Gemeinschaft sind sie, wie z.B. Austrazismen, Belege für den regionalen Reichtum einer Sprache. Das schließt nicht aus, dass ungebildete Rezipienten in solchen SZ 1 "Fehler" erkennen und diese zu sanktionieren versuchen.

"Zweifel" setzen Zweifelnde, also Subjekte des Zweifels, voraus! In der Regel ist das Subjekt eines "sprachlichen Zweifels" ein Individuum. Aber natürlich: Auch ganze Sprecher/ Schreibergruppen können zweifeln (wie die Rechtschreibreform recht gut zeigt). Das gilt im vollen Sinne aber nur für SZ 1. Zweifel von Sprachwissenschaftlern und Linguistinnen im Hinblick auf das, was man sprachstrukturell im Deutschen (noch) sagen kann, sind hingegen sozusagen "objektive Zweifel". Sie spiegeln Probleme von native speaker wider, problematisieren also das "tacit knowledge" im Hinblick auf Grammatikalität/ Ungrammatikalität.

"Sprachliche Zweifel" des Typs SZ 1 setzen hingegen die Bewusstwerdung oder das Bewusstsein von begrenztem sprachlichen Wissen im Sprachgebrauch voraus. D.h.: Sprecher/ Schreiberinnen müssen sich über eigenes fehlendes, eingeschränktes oder unsicheres Wissen bewusst werden. Insofern setzt ein solcher "Zweifel" ein gewisses Bewusstsein über eigene Wissensdefizienzen voraus. ${ }^{13}$

Zweifel des Typs SZ 1 basieren nicht nur auf grammatischen Regeln oder kommunikativen Normen (etwa der Orthographie). Sie basieren ebenso auf Werturteilen: D.h. Zweifel entstehen vielfach hinsichtlich der sozialen, stilistischen oder der ästhetischen Angemessenheit und Akzeptanz einer Form oder Struktur in einer bestimmten Sprachgemeinschaft. Hier geht es darum, unter mehreren Möglichkeiten eine gewünschte, wirkungsvolle oder angemessene Variante auszuwählen oder zuerst zu entwickeln.

"Sprachliche Zweifel" setzen schließlich ein gewisses Maß an verlässlichem Wissen voraus! Fehler können daher ebenso wenig zu sprachlichen Zweifelsfällen führen wie sozusagen ungeregelte Formen oder Strukturen. Insofern gibt es bei potenziellen SZ 2 durchaus Parallelen zu Versprechern oder bestimmten aphasischen Abweichungen, die in ihrer Abweichung jedoch geregelte Strukturen durchscheinen lassen. Nicht jede beliebige sprachliche Form oder Struktur kann zu einem "Zweifelsfall" werden. Denn auch abweichende Formen und Strukturen müssen zumindest einige Analogien zu korrekten Formen aufweisen, d.h. sie dürfen nicht gänzlich regellos sein! Hier gilt die Erkenntnis der sog. "Fehlerlinguistik": Auch z.B. Versprecher können nicht jedwede Form haben, sondern sind trotz ihrer Abweichung ebenfalls partiell regelhaft!

Ein letzter Unterschied zwischen SZ 1 und SZ 2: Sprachliche Zweifel des ersten Typs werden mit dem Ziel reflektiert, diese zu beseitigen.

13 Deshalb können Ignoranten, Dumme oder Stolze auch nicht zweifeln. Ihnen fehlt von Natur aus oder vorhandenes Wissen selbstbewusst ignorierend - gerade jenes Wissen, das sie benötigten, um Lücken, Unklarheiten oder Grenzen überhaupt erst erkennen zu können. Zugespitzt ausgedrückt: Sie wissen nicht nur nichts, sondern sie wissen - anders als Sokrates - auch nicht, dass sie nichts wissen! 
Auf diesem Hintergrund lassen sich SZ 1 wie folgt definieren: Eine bestimmte sprachliche Form oder Struktur soll dann als ein "Zweifelsfall" verstanden werden, wenn

es in einer komplexen Sprachgemeinschaft historische, regionale, grammatische, stilistische, orthographische usw. Varianten oder noch "ungefestigte" Übernahmen, Neukreationen usw. gibt (= notwendige Bedingung) und wenn es ferner

Sprecher/Schreiber-(Gruppen) gibt, die ein Bewusstsein über ein eingeschränktes, ein unsicheres oder gar ein fehlendes sprachlich-kommunikatives Wissen hinsichtlich der unter 1. genannten sprachlichen Formen oder Strukturen entwickeln, mit dem Ziel, diese Wissensdefizienzen zu beseitigen (= hinreichende Bedingung). "Sprachliche Zweifel" erfordern anders als "sprachliches Wissen (im Sinne von Chomsky, vgl. Felix/Fanselow 1990) einen zumindest partiell selbstreflexiven Zugang zu eigenen bewusstseinsfähigen Wissensbeständen.

\section{$5 \quad$ "Sprachliche Zweifelsfälle" und Sprachwandel}

Zum Schluss noch eine vielleicht überraschende Frage: Worin besteht sozusagen der sprachtheoretische Sinn von "sprachlichen Zweifelsfällen"? Wie lassen sich aus dieser Sicht ihre Massivität ebenso wie ihre bereichsspezifischen Ausdifferenzierungen erklären?

In einer evolutionären Linguistik sind solche Fragen vergleichsweise naheliegend. "Sprachliche Zweifelsfälle" sind - so wurde schon angedeutet - eine der wichtigen Bedingungen für sprachliche Dynamik und damit letztlich für Sprachwandel.

Wie hat man sich diesen Prozess vorzustellen? Zunächst sind "sprachliche Zweifelsfälle" bei aller Differenzierung von Spracherwerb und Sprachwandel (vgl. Knobloch 1999) sozusagen "Mutanten" im Prozess der Vererbung sprachlicher Formen und Strukturen in kommunikativen Prozessen (sensu Feilke 1994, 1996, Keller 1994). Sie sind Indikatoren für bewusst empfundene oder auch thematisierte sprachliche, stilistische und ähnliche Konkurrenzformen. Setzt sich eine der Konkurrenzformen durch, dann besteht die Möglichkeit, dass einige dieser Mutanten - sozusagen "bei ein bisschen Glück" - zukünftige Sprachformen bestimmen. Natürlich: Die absolut meisten individuellen Fehler oder Neukreationen bleiben idiosynkratischer Natur. Aber manche werden von anderen Kommunikationsteilnehmern übernommen. Einige wenige davon verfestigen sich dann zum Beispiel als "sprachliche Zweifelsfälle". Wiederum ein Bruchteil obsiegt schließlich gegenüber alternativen Konkurrenten und etabliert sich sprachhistorisch als eine zukünftig erfolgreiche Form oder Struktur. Bei perfektem Wissen aller Sprachteilnehmer könnte ein solcher nicht hundertprozentiger Vererbungseffekt überhaupt nicht auftreten. Oder anders: Nur bei Annahme eines nicht immer perfekten sprachlich-kommunikativen Wissens können überhaupt "sprachliche Zweifelsfälle" entstehen. Insofern ist "Imperfektibilität" ein zentrales synchrones Phänomen, gleichsam eine Zwischenstation in einem in die Zukunft gerichteten diachronen Prozess der Vererbung sprachlicher Formen und Strukturen. Fazit: Unter sprachevolutionärer Perspektive ist "die sich in Zweifeln verkörpernde sprachliche Inkompetenz (...) nicht als defizientes Gegenstück zur traditionellen Sprachkompetenz zu begreifen, sondern als deren gesteigerte Form" (Klein 2003a). 


\section{Literaturangaben}

Antos, Gerd (1996): Laien-Linguistik. Studien zu Sprach- und Kommunikationsproblemen im Alltag. Am Beispiel von Sprachratgebern und Kommunikationstrainings. Tübingen.

Biere, Bernd Ulrich/Hoberg, Rudolf (eds.) (1995): Bewertungskriterien in der Sprachberatung. Tübingen.

Bünting, Karl-Dieter/Pospiech, Ulrike (1996): "Das Sprachtelefon: Beobachtungen von Unsicherheiten und Unzufriedenheiten von Sprachteilhabern ein Werkstattbericht". In: Böke, Karin/Jung, Matthias/Wengeler, Martin (eds.): Öffentlicher Sprachgebrauch. Praktische, theoretische und historische Perspektiven. Georg Stötzel zum 60. Geburtstag. Opladen: 121-127.

Chomsky, Noam (1984): Knowledge of Language: Its Nature, Origin, and Use. New York.

Duden. Richtiges und gutes Deutsch. Wörterbuch der sprachlichen Zweifelsfälle (Bd. 9). 5. neu bearbeitete Auflage. Herausgegeben von der Dudenredaktion. Mannheim. (vormals: Die Zweifelsfälle der deutschen Sprache. Wörterbuch der sprachlichen Hauptschwierigkeiten. Bibliographisches Institut Mannheim 1972)

Fanselow, Gisbert/Felix, Sascha (1990): Sprachtheorie I. Grundlagen und Zielsetzungen. Tübingen.

Feilke, Helmuth (1994): Common sense - Kompetenz. Überlegungen zu einer Theorie des "sympathischen" und "natürlichen" Meinens und Verstehens. Frankfurt/M.

Feilke, Helmuth (1996): Sprache als soziale Gestalt. Ausdruck, Prägung und die Ordnung der sprachlichen Typik. Frankfurt/M.

Höhne, Steffen (1990): "Kommunikationsberatung Deutsch als Fremdsprache". Sprache und Literatur in Wissenschaft und Unterricht 65: 84-95.

Höhne, Steffen (1991): "Sprachnorm und Sprachnormwandel als konstitutive Faktoren wissenschaftlicher Sprachberatung". Muttersprache 101: 193-217.

Hopper, Paul (1987): "Emergent Grammar". Berkeley Linguistics Conference (BLS) 13: 139157.

Ickler, Theodore (2000): Das Rechtschreibwörterbuch: Sinnvoll schreiben, trennen Zeichen setzen. St. Goar.

Kilian, Jörg (2003): "Wörter im Zweifel. Ansätze einer linguistisch begründeten kritischen Semantik". Linguistik-online 16/2003: 159-170.

http:/www.linguistik-online.de/16_03/kilian.html bzw. http:/www.linguistik-online.de/16_03/kilian.pdf.

Klein, Wolf Peter (2003a): Zur Einführung: Sprachliche Zweifelsfälle als linguistischer Gegenstand. Abstract auf der DGfS-Tagung München 2003 AG 2.

Klein, Wolf Peter (2003b): "In dubio contra reum? Zur Geschichte der Reflexion über sprachliche Zweifelsfälle". Tribüne 2/2003: 4-14.

Knobloch, Clemens (2000): Spracherwerb und Sprachwandel: Zweckehe oder gefährliche Liebschaft? Siegen. 
Schmitz, Ulrich (2000) "AUSFAHRT waschen. Über den progressiven Untergang der Flexionsfähigkeit". Sprache an der Jahrtausendwende. Osnabrücker Beiträge zur Sprachtheorie, H. 60: 135-182.

http://www.linse.uni-essen.de/linse/publikationen/ausfahrt_waschen.html

Weber, Tilo (1997): "The emergence of linguistic structure: Paul Hopper's emergent grammar hypothesis revisited". Language Science 19/2: 177-196. 